\title{
First Principles Calculations of Shock Compressed Fluid Helium
}

\author{
B. Militzer \\ Geophysical Laboratory, Carnegie Institution of Washington, \\ 5251 Broad Branch Road, NW, Washington, DC 20015
}

(Dated: October 26, 2018)

\begin{abstract}
The properties of hot dense helium at megabar pressures were studied with two first-principles computer simulation techniques, path integral Monte Carlo and density functional molecular dynamics. The simulations predicted that the compressibility of helium is substantially increased by electronic excitations that are present in the hot fluid at thermodynamic equilibrium. A maximum compression ratio of 5.24(4)-fold the initial density was predicted for $360 \mathrm{GPa}$ and $150000 \mathrm{~K}$. This result distinguishes helium from deuterium, for which simulations predicted a maximum compression ratio of 4.3(1). Hugoniot curves for statically precompressed samples are also discussed.
\end{abstract}

There has been considerable controversy in the deuterium equation of state (EOS) since laser shock wave experiments probed the megabar pressure regime for the first time and predicted that deuterium is highly compressible under shock conditions to approximately 6-fold the initial density 1, 2]. Such a high compression ratio was neither reproduced with magnetic compression experiments $[3,4]$ nor with explosively driven shocks [5, 6 ]. Both sets of later experiments predicted compression ratios close to 4.3(1), which is in good agreement with results from first principles computer simulations $7,6,8,[$. When we applied the same simulation techniques, path integral Monte Carlo (PIMC) and density functional molecular dynamics (DFT-MD), to hot dense helium, we found that helium's shock compressibility is substantially increased due to electronic excitations in the fluid.

In this Letter, we make the prediction that electronic excitations in helium lead to a maximum shock compression ratio of 5.24(4), while such excitations in deuterium do not increase the compressibility ratio beyond 4.3(1). Furthermore, we show that the compression ratio is reduced when the sample is precompressed statically in a diamond anvil cell before a shock is launched. Such novel compression techniques are currently developed and data for dense helium are forthcoming [10]. The combination of static and dynamic compression techniques allows the study of materials at much higher densities, and their application to hydrogen and helium will enable a direct characterization of a much larger section of the isentrope that determines the interiors of giant planets.

Present studies of giant planetary interiors 11] are based on approximate free energy models that rely on analytical thermodynamic expressions and are often fit to experimental results if available. Although these models (for helium read 12, 13, 14]) are very practical, their predictive capabilities for shock states and the EOS are limited because interaction and polarization effects in a dense fluid are very difficult to study analytically, which underlines the need of first principles simulations.

Shock wave experiments provide us with direct information for materials' EOS at high pressure and temperature. When a shock wave passes through the sample, the thermodynamic state of the material, characterized by the internal energy, pressure, and volume, changes from initially $\left(E_{0}, P_{0}, V_{0}\right)$ to the final values of $(E, P, V)$. The conservation of mass, momentum, and energy yields the Hugoniot condition [15],

$$
H=\left(E-E_{0}\right)+\frac{1}{2}\left(P+P_{0}\right)\left(V-V_{0}\right)=0 .
$$

The resulting Hugoniot curve is the locus of all final states that can be reached for different shock velocities. Theoretically, the Hugoniot curve can be calculated from the EOS, assuming $P_{0} \ll P$ and using the initial molecular volume from the experiment, $V_{0}=32.4 \mathrm{~cm}^{3} / \mathrm{mol}$ $\left(\rho_{0}=0.1235 \mathrm{~g} \mathrm{~cm}^{-3}\right)$ [16]. For $E_{0}$ one takes the energy of an isolated helium atom, which must be consistent with the internal energy $E(V, T)$ derived from a particular method. For PIMC we use $E_{0}=-79.0048 \mathrm{eV}$ per atom because PIMC is exact for the helium atom. The assumptions for $P_{0}$ and $E_{0}$ also remain sufficiently valid for samples that have been precompressed statically to 4 times $\rho_{0}$.

PIMC is a finite-temperature quantum simulation method that we used to model dense fluid helium as a system of nuclei and electrons that interact via the Coulomb potential. Both types of particles are explicitly treated as paths and all correlation effects are included, which makes PIMC one of the most accurate finite-temperature quantum simulation methods available. The only noncontrolled approximation required is the fixed node approximation that is introduced to treat the fermion sign problem, which arises from the explicit treatment of electrons. The fermion nodes are taken from a thermal trial density matrix, for which we extended the variational density matrix approach [17] to helium.

We complement our PIMC EOS with DFT-MD data, since DFT is much more efficient at low temperatures because it is in principle a ground state electronic structure method. The DFT-MD trajectories were obtained with Born-Oppenheimer MD where the electrons were assumed to be in the instantaneous ground state. We used the CPMD code [18 using the PBE generalized gradient approximation [19] with $N=64$ atoms, a time step of 


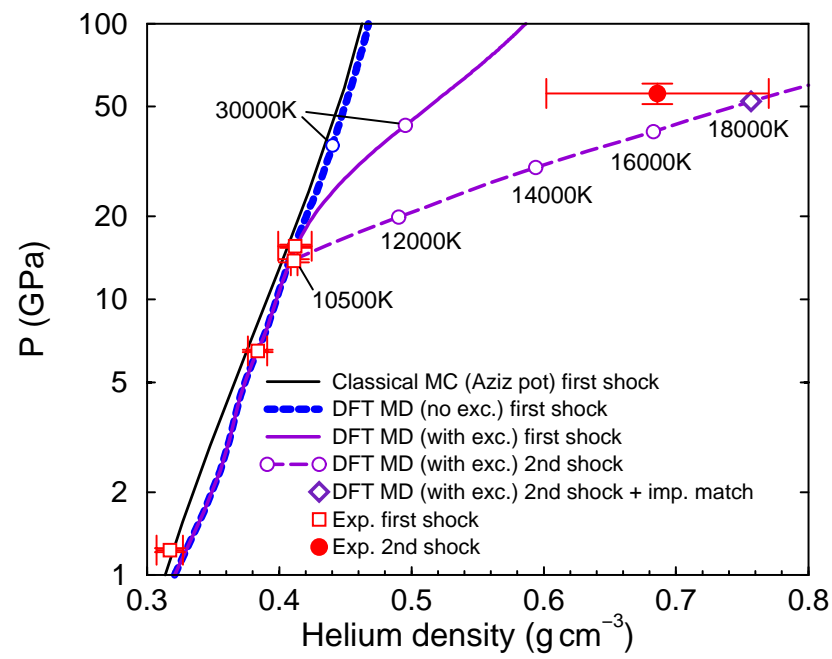

FIG. 1: The principal shock Hugoniot curves computed using DFT-MD with (solid line) and without (blue dashed line) the consideration of excited electronic states are compared with gas gun shock wave experiments [16] (open red symbols). The dashed purple line shows the computed reshock curve. The $\diamond$ on it indicates the impedance match condition for the reshock experiment [16] (solid red symbol).

$0.77 \mathrm{fs}$, Troullier-Martin pseudopotentials, and a $100 \mathrm{Ry}$ cut-off for the plane wave expansion of the Kohn Sham orbitals, combined with $\Gamma$ point sampling of the Brillioun zone. A finite size study showed that the Hugoniot results are well converged with $N=64$.

The only available shock data for fluid helium were obtained with gas gun experiments by Nellis et al. 16]. The comparison shown in Fig. [1 shows excellent agreement between experimental data and DFT-MD simulation results for the principal and the reshock Hugoniot curves.

The DFT-MD results without electronic excitations are closely tracked by data from classical Monte Carlo (CMC) simulations using the Aziz pair potential [20]. This potential was derived to describe the interaction of two isolated helium atoms. With rising shock pressure, DFT-MD and CMC curves in Fig. 2 show a gradual increase in compression towards 4-fold the initial density, which represents the high pressure limit, where the kinetic energy dominates over the potential energy and the system behaves approximately like non-interacting particles.

Conversely, the results from PIMC calculations predict much higher compression ratios, reaching a maximum of $5.24 \pm 0.04$ for $P=360 \mathrm{GPa}$ and $T=150000 \mathrm{~K}$. This increase in compression is due to electronic excitations that are present in the hot dense fluid at thermodynamic equilibrium. Helium differs in this regard from shocked deuterium, for which PIMC calculation predicted a maximum compression ratio of only 4.3(1) [8].

In our PIMC simulation program, the pair density ma-

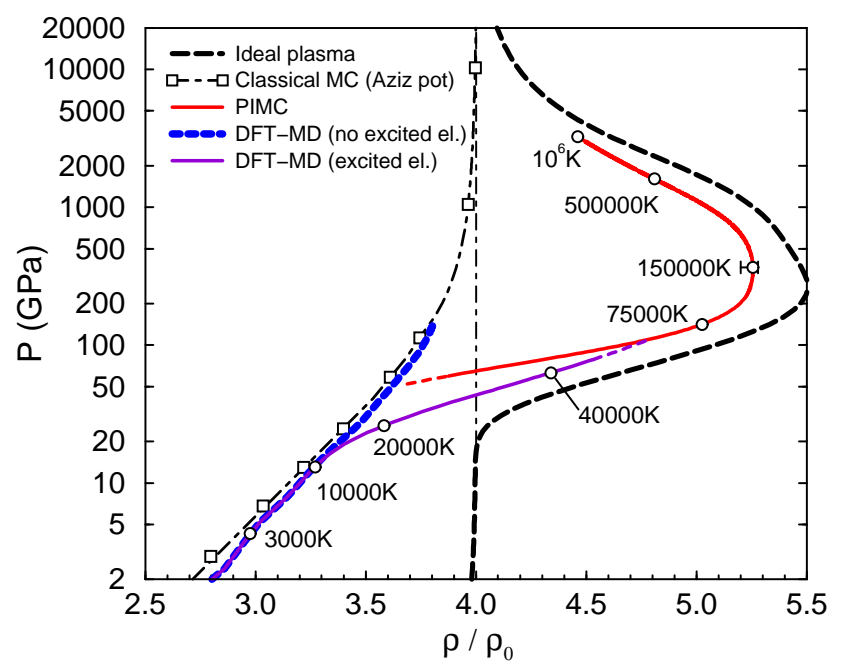

FIG. 2: The principal shock Hugoniot curve of helium is shown as a function of compression ratio. PIMC and DFTMD results were combined to span a large range of temperature, as the circles in the curve indicate. Effects of thermal electronic excitations (purple solid line) were used to correct the ground state DFT-MD calculations (blue dashed line), which resulted in a strong increase in compressibility. Results a from classical simulation using the Aziz pair potential and from a non-interacting plasma model are included.

trix technique 21, 22] is employed to treat the Coulomb interactions. The accurate treatment of the many-body correlations and the application of the nodal restriction for the paths requires the use of a small step, $\tau=\beta / M$, to discretize the paths in imaginary time $\beta=1 / k_{B} T$ into $M$ steps. Using a time step between $\tau^{-1}=2 \times 10^{6}$ and $16 \times 10^{6} \mathrm{~K}$ depending on density, allowed a reduction of the remaining time step error in the observables below the size of MC error bars.

PIMC simulations with $N_{e}=32$ electrons and $N=16$ nuclei were performed on a grid of 12 densities and 5 temperatures ranging from $3.5 \geq r_{s} \geq 1.0$ and $10^{6} \geq T \geq$ $61250 \mathrm{~K}$. The resulting EOS was interpolated to obtain the Hugoniot curves. A finite-size extrapolation with up to $N=57$ nuclei was done for four points in $T$ - $\rho$ space, $T=125000$ and $10^{6} \mathrm{~K}$ combined with $r_{s}=1.86$ and 1.25 . Above $100 \mathrm{GPa}$, the Hugoniot curve is insensitive to finite size corrections because of the high temperatures and due to the partial cancellation of pressure and internal energy corrections in Eq. 1 After propagating the correction and the uncertainty of the finite size extrapolation, we obtained $5.24 \pm 0.04$ as maximum compression ratio of fluid helium, which actually brackets the original value of 5.25 obtained for $N=16$.

The predicted increase in compression beyond 4 -fold the initial density in helium can be understood by invoking a very simple free energy model, which assumes helium to be composed of different non-interacting species, $\mathrm{He}, \mathrm{He}^{+}$, and $\mathrm{He}^{2+}$, to represent the various ionization 
stages of helium as well as free electrons. The resulting Hugoniot curve shows a similar increase in the compression ratio demonstrating that the increase beyond 4 -fold is due to electronic excitations leading to free electrons.

To further verify this hypothesis, we corrected the DFT EOS for finite-temperature electronic effects. For a number of snapshots along the MD trajectory, we thermally populated the instantaneous excited electronic states [23] using the Mermin functional with up to 7 additional orbitals per atom. For temperatures above $15000 \mathrm{~K}$, the resulting corrections to the internal energy and pressure leads to a substantial increase in shock compressibility. This increase is primarily caused by a rise in the internal energy due to thermal population of excited electronic states. Fluid helium maintains a wide excitation gap ranging between 5 and $15 \mathrm{eV}$ for $T \leq 80000 \mathrm{~K}$ and $2.6 \geq r_{s} \geq 1.0$. On the principal Hugoniot, electronic excitations occur above $20 \mathrm{GPa}$, which explains why the gas gun experiments have not reached the regime of electronic excitations. Even a gas gun reshock experiment would be insufficient because the final temperatures remain relatively low (Fig. 1), and facilities that can generate faster shock waves are needed instead.

The maximum shock pressures that can be reached at a particular experimental facility depend on the power of the drive but also on the impedance of the sample material. For the same shock drive, the final shock pressure is, to a first approximation, proportional to the initial density of the sample material. The maximum pressure reported for deuterium Nova laser shocks 1, 2], $340 \mathrm{GPa}$, and the highest achievable on the $\mathrm{Z}$ machine [3, 4], 175 $\mathrm{GPa}$, would translate to approximately $246 \mathrm{GPa}$, and $127 \mathrm{GPa}$ 28] in helium. Consequently, with both facilities one would be able reach the regime of the predicted 5 -fold compression and probe the effect of electronic excitations.

The comparison in Fig. 3 shows the discussed increase in compressibility beyond 4-fold for helium, while our results for deuterium [8, 26] show hardly any, despite the similarity of the two fluids. Deuterium molecules and helium atoms have the same mass and provide two mechanisms to absorb shock energy, which in principle can lead to shock compression ratios substantially larger than 4 . The helium atom has two ionization stages with energies of 24.6 and $54.4 \mathrm{eV}$. Deuterium molecules dissociate with $4.5 \mathrm{eV}$ energy, and the ionization of resulting atoms requires $13.6 \mathrm{eV}$. However, the explanation for the different shock behavior of helium and deuterium is not a consequence of single particle properties but is a result of different degrees of particle interaction. Fig. 4 shows the Hugoniot function, $H$, for both materials at 5-fold compression. For helium, first principles calculation that include the interaction effects, as well as the non-interacting plasma model, predict that $H$ changes its sign, implying that helium is more than 5-fold compressible. While the non-interacting plasma model predicts

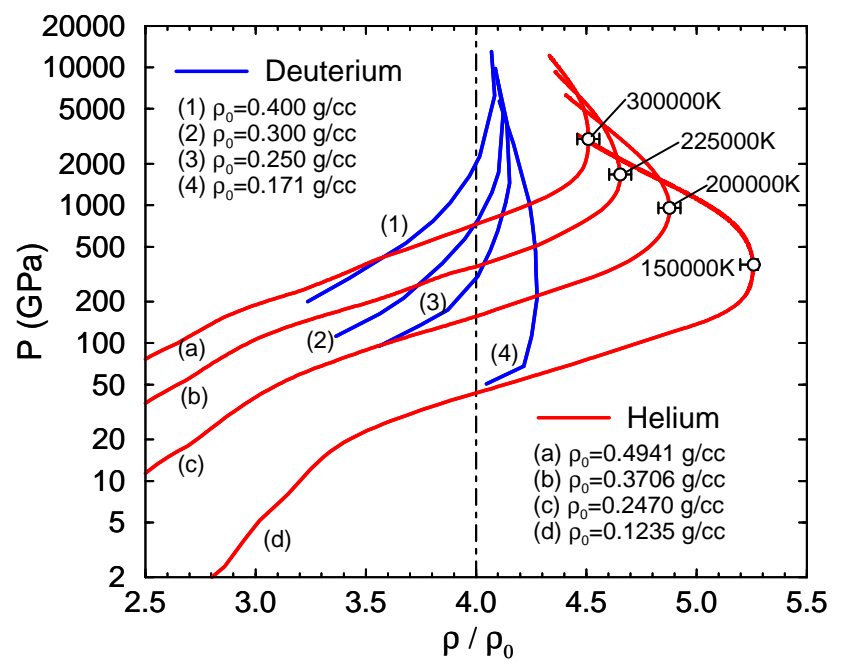

FIG. 3: The principal shock Hugoniot curves for deuterium [24] and helium are shown for samples that were precompressed to different initial densities. For both materials, the precompression reduces the maximum compression ratio $\rho / \rho_{0}$ that can be reached. For helium, the indicated initial densities (a)-(d) correspond to the initial pressures of $7.1 \mathrm{kbar}$, $1.8 \mathrm{kbar}, 188$ bar [25], and 1 bar. The DFT-MD and PIMC data shown in Fig. 2 were interpolated for helium.

the same behavior also for deuterium, the PIMC simulations show that the $H$ function is strictly negative because the pressure is substantially higher than suggested by the non-interacting plasma model.

The interaction effects at 5-fold compression are stronger in deuterium than in helium because in helium, the initial density is lower and, more importantly, the electronic orbitals are much more localized. The initial density, $\rho_{0}=0.1235 \mathrm{~g} \mathrm{~cm}^{-3}$ corresponds to a WignerSeitz radius of $r_{s}=3.51\left[\frac{4}{3} \pi\left(r_{s} a_{0}\right)^{3}=V / N_{e}\right]$, and $95 \%$ of the electron charge is localized in $10 \%$ of the total volume. The initial density of fluid deuterium, $\rho_{0}=0.171 \mathrm{~g} \mathrm{~cm}^{-3}$ corresponds to $r_{s}=3.16$, and $95 \%$ of the charge is localized in $40 \%$ of the volume. With increasing compression, the orbitals in deuterium start to overlap sooner and the resulting polarization and exchange interactions will increase the pressure, which causes deuterium to appear less compressible in shock experiments.

In Fig. 3] we use our first principles calculations to predict how the static precompression will affect the Hugoniot curves of helium and deuterium 24]. For helium, a precompression to 4-fold the cryogenic liquid density at 1 bar, $\rho_{0}=4 \rho_{0}^{(1 \mathrm{bar})}$, increases the maximum density on the Hugoniot curve to $\rho=17.5 \rho_{0}^{(1 \text { bar })}$. However, the maximum compression ratio for the dynamic compression, $\eta=\rho / \rho_{0}$, decreases in both materials with increasing precompression. The increase in the initial density leads to stronger interactions under shock conditions. In general the shock compression ratio is determined by the relative importance of excitations of internal degrees of 

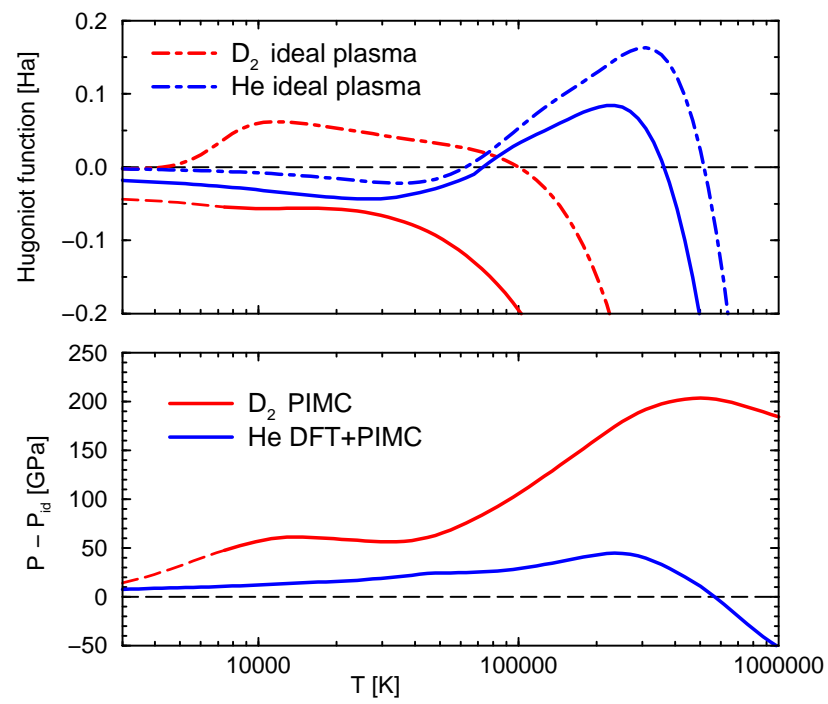

FIG. 4: The Hugoniot functions $H$ from Eq. 1for helium and deuterium derived from first principles calculation (solid lines) and from the non-interacting plasma model (dashed line) is shown at 5-fold compression. Deuterium is less compressible than helium because the interaction between the particles is much stronger, which leads to a substantial increase in pressure beyond the corresponding value of the non-interacting system including ionization $\left(P_{\mathrm{id}}\right)$, illustrated by the pressure difference in the lower graph.

freedom that increase the internal energy and interaction effects that increase the pressure.

The point of maximum compression, $\eta_{\max }$, along the Hugoniot curve is reached when the Grüneisen parameter, $\left.\gamma \equiv V \frac{\partial P}{\partial E}\right|_{V}$, satisfies $\gamma=\frac{2}{\eta-1}$. A reduction in the compression ratio with precompression can be expressed by $\frac{d \eta_{\max }}{d V_{0}}>0$. Using Eq. 1 and assuming $E_{0}$ does not change with the precompression, this condition can be expressed in terms of the simple thermodynamic condition, $\left.\frac{\rho}{P} \frac{\partial P}{\partial \rho}\right|_{E}>1$. We have computed the isoenergetic compressibility, $\left.\frac{\partial P}{\partial \rho}\right|_{E}$, and verified that this condition is satisfied for hydrogen and helium by using our first principles results as well as with the non-interacting plasma model. This confirms that the precompression reduces the maximum compression ratio in hydrogen and helium, and one can postulate that this might also be true for other simple fluids.

The computed EOS shows that helium and hydrogen behave differently at high pressure, which has important implications for the interior structure of solar and extrasolar giant planets where it is has been predicted that the two fluid phases become immiscible at high pressures [27]. Our calculations predict that helium is 5.24(4)-fold compressible under shock conditions, which distinguishes it from fluid deuterium, for which first principles calculations and recent experiments [3, 4, 5, 6] predicted a maximum compression ratio close to 4.3. We suggest that all deuterium experiments be repeated with helium in order to validate the different shock compression techniques. This validation is important before the EOS can be used for wide range of applications including the modeling of giant planets and to draw conclusions about their evolution.

The author acknowledges fruitful discussions with $\mathrm{N}$. Ashcroft, J.D. Johnson, R. Hemley, R. Cohen, E.L. Pollock, S. Gramsch, I. Tamblyn, and J. Vorberger. This material is based upon work supported by NASA under the grant NNG05GH29G and by the NSF under the grant 0507321 .

[1] L. B. Da Silva et al. Phys. Rev. Lett., 78:483, 1997.

[2] G. W. Collins et al. Science, 281:1178, 1998.

[3] M. D. Knudson et al. Phys. Rev. Lett., 87:225501, 2001.

[4] M. D. Knudson et al. Phys. Rev. Lett., 90:035505, 2003.

[5] S.I. Belov et al. JETP Lett., 76:443, 2002.

[6] G. V. Boriskov et al. Phys. Rev. B, 71:092104, 2005.

[7] T. J. Lenosky, J. D. Kress, and L. A. Collins. Phys. Rev. $B, 56: 5164,1997$.

[8] B. Militzer and D. M. Ceperley. Phys. Rev. Lett., 85:1890, 2000.

[9] S. A. Bonev, B. Militzer, and G. Galli. Phys. Rev. B, 69:014101, 2004.

[10] P. Loubeyre et al., paper presented at the meeting of the American Physical Society, March 21-25, 2005, Los Angeles, CA.

[11] T. Guillot el al., in Jupiter, F. Bagenal, Ed. (Univ. of Arizona Press, Tucson, 2003), chap. 3, pp. 35-57.

[12] D. Saumon, G. Chabrier, and H. M. Van Horn. Astrophys. J. Suppl., 99:713, 1995.

[13] V. Schwarz, H. Juranek, and R. Redmer. Phys. Chem. Chem. Phys., 7:1990, 2005.

[14] C. Winisdoerffer and G. Chabrier. Phys. Rev. E, 71:026402, 2005.

[15] Y. B. Zeldovich and Y. P. Raizer. Academic Press, New York, 1966.

[16] W. J. Nellis et al. Phys. Rev. Lett., 53:1248, 1984.

[17] B. Militzer and E. L. Pollock. Phys. Rev. E, 61:3470, 2000.

[18] CPMD, Copyright IBM Corp 1990-2006, MPI für Festkörperforschung Stuttgart 1997-2001.

[19] J. P. Perdew, K. Burke, and M. Ernzerhof. Phys. Rev. Lett., 77:3865, 1996.

[20] R.A. Aziz, A.R. Janzen, and M. R. Moldover. Phys. Rev. Lett., 74:1586, 1995.

[21] E.L. Pollock and D. M. Ceperley. Phys. Rev. B, 30:2555, 1984.

[22] B. Militzer and R. L. Graham. J. Phys. Chem. Solids, 67:2143, 2006.

[23] Copyright ABINIT group (M. Mikami, J. M. Beuken, X. Gonze) 2003-2005.

[24] B. Militzer. J. Phys. A: Math. Gen., 63:6159, 2003.

[25] A. Driessen, E. van der Poll, and I. F. Silvera. Phys. Rev. B, 33:3269, 1986.

[26] B. Militzer et al. Phys. Rev. Lett., 87:275502, 2001.

[27] D.J. Stevenson and E.E. Salpeter. Astrophys. J. Suppl. Ser., 35:221, 1977.

[28] A more accurate estimate for $\mathrm{Z}$ machine including the 
impedance match yields $140 \mathrm{GPa}$. (M. Knudson, private communication.) 